\title{
Hypervariable intronic region in NCX1 is enriched in short insertion-deletion polymorphisms and showed association with cardiovascular traits
}

\author{
Katrin Kepp', Elin Org ${ }^{1}$, Siim Sõber ${ }^{1}$, Piret Kelgo ${ }^{1}$, Margus Viigimaa ${ }^{2,3}$, Gudrun Veldre ${ }^{1,4}$, Neeme Tõnisson ${ }^{1}$,
} Peeter Juhanson', Margus Putku', Andreas Kindmark', Viktor Kožich', Maris Laan ${ }^{1 *}$

\begin{abstract}
Background: Conserved non-coding regions (CNR) have been shown to harbor gene expression regulatory elements. Genetic variations in these regions may potentially contribute to complex disease susceptibility.

Methods: We targeted CNRs of cardiovascular disease (CVD) candidate gene, $\mathrm{Na}(+)-\mathrm{Ca}(2+)$ exchanger (NCX1) with polymorphism screening among CVD patients $(n=46)$ using DHPLC technology. The flanking region (348 bp) of the $14 \mathrm{bp}$ indel in intron 2 was further genotyped by DGGE assay in two Eastern-European CVD samples: essential hypertension (HYPEST; 470 cases, 652 controls) and coronary artery disease, CAD (CADCZ; 257 cases, controls 413). Genotype-phenotype associations were tested by regression analysis implemented in PLINK. Alignments of primate sequences were performed by ClustalW2.

Results: Nine of the identified NCX1 variants were either singletons or targeted by commercial platforms. The 14 bp intronic indel (rs 11274804) was represented with substantial frequency in HYPEST (6.82\%) and CADCZ (14.58\%). Genotyping in Eastern-Europeans $(n=1792)$ revealed hypervariable nature of this locus, represented by seven alternative alleles. The alignments of human-chimpanzee-macaque sequences showed that the major human variant (allele frequency 90.45\%) was actually a human-specific deletion compared to other primates. In humans, this deletion was surrounded by other short (5-43 bp) deletion variants and a duplication (40 bp) polymorphism possessing overlapping breakpoints. This indicates a potential indel hotspot, triggered by the initial deletion in human lineage. An association was detected between the carrier status of $14 \mathrm{bp}$ indel ancestral allele and CAD ( $P$ $=0.0016, \mathrm{OR}=2.02$; Bonferroni significance level alpha $=0.0045)$, but not with hypertension. The risk for the CAD development was even higher among the patients additionally diagnosed with metabolic syndrome $(P=0.0014$, $\mathrm{OR}=2.34$ ). Consistent with the effect on metabolic processes, suggestive evidence for the association with heart rate, serum triglyceride and LDL levels was detected $(P=0.04)$.
\end{abstract}

Conclusions: Compared to SNPs targeted by large number of locus-specific and genome-wide assays, considerably less attention has been paid to short indel variants in the human genome. The data of genome dynamics, mutation rate and population genetics of short indels, as well as their impact on gene expressional profile and human disease susceptibility is limited. The characterization of NCX1 intronic hypervariable non-coding region enriched in human-specific indel variants contributes to this gap of knowledge.

\footnotetext{
* Correspondence: maris@ebc.ee

${ }^{1}$ Institute of Molecular and Cell Biology, University of Tartu, Tartu, Estonia
} 


\section{Background}

Cardiovascular disease (CVD) is a complex disorder affecting heart and blood vessels, which develops from the interaction between life style patterns and genetic susceptibility to the disease. Western societies face high and increasing rates of CVD (such as coronary artery disease, hypertension, arteriosclerosis, heart failure and arrhytmia etc.), which is considered a number one cause of premature death and disability. Although CVD has been shown to have significant heritability, pinpointing of the genes and variants associated with the elevated risk to the disease has been challenging $[1,2]$. The focus has slowly switched from DNA variants located in genic regions causing direct changes in the encoded protein to the regulatory variants affecting gene expression. Noncoding variants potentially contributing to the susceptibility to complex diseases are localized in promoters and enhancers, introns or 5'- and 3'-UTRs, and may affect binding of the gene expression regulators, such as transcription and splicing factors or miRNAs. Comparative genetics studies have noted several essential gene regulatory elements that are conserved among species $[3,4]$. Thus, targeting evolutionarily conserved non-coding regions (CNR) in candidate genes for CVD may pinpoint regulatory elements directing the gene expression profile. Genetic variation in these regions may contribute to the susceptibility to CVD. Based on these hypotheses we aimed to target human CVD candidate gene $\mathrm{Na}(+)-\mathrm{Ca}(2+)$ exchanger (NCX1; SLC8A1) with polymorphism screening in CNRs and to test associations of identified variants with CVD and related metabolic traits in two Eastern-European populations.

$\mathrm{Na}^{+} / \mathrm{Ca}^{2+}$ exchange participates in the regulation of vascular function and thus, disturbances in this process contribute to the development of CVD. $\mathrm{Na}^{+} / \mathrm{Ca}^{+2}$ exchanger (NCX1) is a bidirectional calcium transporter, responsible for calcium homeostasis in cardiac myocytes and in other cell types by catalyzing the exchange of one $\mathrm{Ca}^{2+}$ ion for three $\mathrm{Na}^{+}$ions across plasma membrane [5]. Altered $\mathrm{Na}^{+} / \mathrm{Ca}^{2+}$ exchange activity has been observed in arrhythmias, heart failure [6], and salt-sensitive essential hypertension $[7,8] . N c x 1^{-1-}$ mice showed complete lack of $\mathrm{Na}^{+} / \mathrm{Ca}^{2+}$ exchange activity in heart leading to the defects in heart development and embryonic lethality [9].

NCX1 gene (498 $908 \mathrm{bp}$ ) is located in chromosome 2p22.1 and consists of 12 alternatively spliced exons[10]. Alternative splicing of $N C X 1$ produces several tissuespecific isoforms [11] differing in their regulatory properties $[12,13]$. NCX1 alternative isoforms respond differently to potential therapeutic agents such as polyunsaturated fatty acids [14] and specific NCX1 inhibitors [9]. Currently, genetic studies targeting the association of $N C X 1$ polymorphisms with CVD are limited. Resequencing of the entire coding and promoter regions in Japanese population identified 15 polymorphisms, two of these variants located $>23 \mathrm{~kb}$ upstream of the mRNA transcription start site were associated with hypertension [8].

We have conducted a polymorphism screening in NCX1 non-coding regions. The most potential genetic variant to affect gene function, a $14 \mathrm{bp}$ indel, localized in an intronic hypervariable region was characterized in detail in cardiovascular and metabolic traits in two European populations.

\section{Methods}

In silico analysis of conserved non-coding regions (CNR) of NCX1

We screened NCX1 (also known as SLC8A1) locus for the presence of Conserved Non-coding Regions (CNRs) using the web-based VISTA software [15] with the proposed default parameters (cutoff criteria: 100 bp sliding window; sequence identity $70 \%$; comparison with rat and mouse). The analyzed NCX1 locus (in total 420,181 bp) spanned from $10 \mathrm{~kb}$ downstream to $10 \mathrm{~kb}$ upstream of the gene [2p22.1; coordinates 40241 046-40 661226 according to NCBI Build 35, hg17; GenBank:6546]. All VISTA regions that had any overlap with annotated genes track at UCSC Genome Browser [16] were excluded as potential coding regions. For polymorphism discovery we selected 29 non-coding regions based on the following criteria: the (i) length 50-300 bp; (ii) location $>200$ bp from the nearest exons, and (iii) sequence identity $>70 \%$ between human and both rodents (See additional file 1).

\section{DHPLC screening of novel polymorphisms in NCX1 non- coding regions}

The selected NCX1 conserved non-coding segments were targeted to polymorphism screening by Denaturing High-Performance Liquid Chromatography method (DHPLC; Wave Technologies Inc. USA). During the design and experimental screening process of DHPLC products the recommendations of the manufacturer were followed. Details of DHPLC assay and running conditions with appropriate PCR primers and fragment characteristics are given in additional file 2. Among the total 29 CNR-s selected, 16 intronic regions entered the DHPLC screening (See additional file 1) phase. 13 regions were excluded before the laboratory experiments due to failure in DHPLC primer design, inappropriate length of the PCR and CNR fragment (too long $>700 \mathrm{bp}$ or short $<50 \mathrm{bp}$ ), or more than two different Tm melting points for the region of interest. The average length of the screened CNR segments was 163 bp (SD: $64 \mathrm{bp}$, 
range: 70-287 bp) and PCR fragments was $334 \mathrm{bp}$ (SD: $87 \mathrm{bp}$, range 170-489 bp). Polymorphism screening was performed with 15 different DNA pools, each consisting of DNAs of three patients with cardiovascular disease originating from two Eastern European sample sets $(\mathrm{n}=$ 22 from HYPEST and $\mathrm{n}=24$ from CADCZ study; detailed description is given below). Individual DNAs in the pools exhibiting evidence for the presence of a polymorphism were sequenced at least twice on both forward and reverse orientations.

\section{DGGE genotyping assay}

Genotyping of the identified 14 bp indel (rs11274804, NCBI dbSNP database) in NCX1 intron 2 was performed by standard Denaturing Gradient Gel Electrophoresis (DGGE) (Ingeny, Goes, Netherlands). The manufacturer's recommendations were followed in the design of the DGGE assay and in choosing the conditions for the experimental setup. Detailed information of the assay is given in additional file 2 . To initially validate the reliability of the DGGE assay, all DNA samples with alternative genotypes previously detected by DHPLC were re-genotyped at the DGGE platform. To further assure DGGE gel typing system's quality in each assay, double positive (product containing $14 \mathrm{bp}$ indel) and negative controls were used. All ambiguous genotypes in DGGE analysis were re-genotyped twice and/or sequenced on both DNA strands by an ABI 377 Prism automated DNA sequencer using ReproGel 377 gels (Amersham Biosciences Inc., USA). The sequences of all novel variants were verified by resequencing twice on both forward and reverse orientation.

\section{Subjects for association studies with cardiovascular disease}

Two Eastern-European sample collection, HYPEST and CADCZ were used to conduct association analysis of rs11274804 with cardiovascular traits and serum biomarkers (Table 1). The HYPEST study has been approved by the Ethics Committee on Human Research of University of Tartu (no. 122/13, 22.12.2003; 137/20, 25.04.2005). CADCZ study was approved by the Ethics Committee of Charles University-1st Faculty of Medicine (December 1996) and is published elsewhere [17]. The studies were carried out in compliance with the Helsinki Declaration and all the participants have given their written informed consent. These sample collections have been recruited to target the genetic-epidemiological component of cardiovascular disease in Estonian and Czech populations, respectively. HYPEST subjects were recruited across Estonia during 2004-2007 (1823 individuals, age range 18-85 years) with the aim to evaluate risk factors for essential hypertension and related cardiovascular disease. Details of the recruitment are given in additional file 2. CADCZ subjects ( $\mathrm{n}=893$; $\mathrm{n}=296$ coronary artery disease patients, $n=597$ controls) were recruited by the Cardiology Department of the $2^{\text {nd }}$ Clinic of Internal Medicine, Faculty Hospital Královské Vinohrady in Prague Czech Republic and Czech heath clinics in years 1998-2000 [17]. In order to exclude obesity and age-related risks, the current study included individuals with BMI $<35 \mathrm{~kg} / \mathrm{m}^{2}$ and age $<65$ years. From HYPEST individuals 470 hypertensive patients and 652 normotensive controls, and from CADCZ samples 257 CAD patients and 413 controls, were analyzed. The control group for both studies consisted of matched healthy individuals with no personal history of CAD, essential hypertension, MI, peripheral arterial disease, or stroke. As no population differentiation was detected among HYPEST and CADCZ study subjects previously [18], the controls of the two studies were pooled in order to address the association of rs11274804 with cardiovascular traits in general Eastern-European population.

\section{Cardiovascular phenotype}

For all subjects in the HYPEST and CADCZ studies resting blood pressure (BP) and heart rate were measured by trained clinicians during recruitment. In both studies BP measurements per subject were obtained after a rest in a sitting position using a standard mercury column sphygmomanometer and size-adjusted cuffs. All HYPEST individuals possessed a documented history of multiple SBP and DBP readings (on average 4.31 readings per individual, range 2-29) during mean 3.17 years (range 1-17 years). To compensate for the variability in heaviness of data per study subjects, we used for the analysis the median across the longitudinal $B P$ readings as well as the median of the subject's age during the readings. Definition of essential hypertension among HYPEST subjects is given in additional file 2 . For CADCZ subjects three blood pressure measurements were documented and the median value was recorded.

Coronary artery disease (CAD) in CADCZ study was diagnosed according to WHO criteria, and one or more large stenosis of a major coronary vessel was confirmed by coronarography in all patients details of which have been published elsewhere [17]. Carotid wall intima media thickness (IMT) and the presence of carotid plaque, recorded in the CADCZ subjects were determined by ultrasonography using linear exploring coil 7,5 $\mathrm{MHz}$ on the distant interior wall about $1-2 \mathrm{~cm}$ distally from the bifurcation. The measurement was performed on the right and the left carotid 5-10 times on each side. Diagnosis of metabolic syndrome was defined based on the criteria appointed by International Diabetes Federation [19]. 
Table 1 Phenotypic characteristics of analyzed individuals

\begin{tabular}{|c|c|c|c|c|c|}
\hline Variable & $\begin{array}{l}\text { HYPEST } \\
\text { Cases }\end{array}$ & Controls & $\begin{array}{l}\text { CADCZ } \\
\text { Cases }\end{array}$ & Controls & $\begin{array}{l}\text { Healthy Eastern } \\
\text { European individuals }{ }^{1}\end{array}$ \\
\hline No. of individuals & 470 & 652 & 257 & 413 & 1065 \\
\hline \multicolumn{6}{|l|}{ Parameters (mean \pm SD): } \\
\hline Age $(\text { years })^{2}$ & $43.9(13.0)$ & $39.0(4.9)$ & $51.2(8.1)$ & $49.5(7.4)$ & $43.7(9.7)$ \\
\hline Body Mass Index (BMI) $\left(\mathrm{kg} / \mathrm{m}^{2}\right)$ & $28.7(3.7)$ & $24.4(3.3)$ & $27.9(3.3)$ & $25.3(3.1)$ & $24.7(3.1)$ \\
\hline Systolic blood pressure (SBP) (mmHg) & $143.2(17.6)$ & $130.0(22.7)$ & $136.6(19.2)$ & $125.2(14.0)$ & $128.5(12.2)$ \\
\hline Diastolic blood pressure (DBP) (mmHg) & $87.4(10.6)$ & $81.0(14.6)$ & $85.1(11.3)$ & $80.3(9.3)$ & $80.0(8.2)$ \\
\hline Total cholesterol (mmol/liter) & $5.6(1.1)$ & $5.0(1.4)$ & $5.4(1.0)$ & $5.6(1.0)$ & $5.4(1.1)$ \\
\hline High-density lipoprotein (HDL) (mmol/liter) & $1.5(0.4)$ & $1.7(0.5)$ & $1.2(0.3)$ & $1.5(0.4)$ & $1.5(0.4)$ \\
\hline Low-density lipoprotein (LDL) (mmol/liter) & $3.8(1.0)$ & $3.3(1.1)$ & $3.2(0.8)$ & $3.4(0.9)$ & $3.3(0.9)$ \\
\hline Triacylglycerols (mmol/liter) & $1.8(1.6)$ & $0.8(0.3)$ & $2.1(1.3)$ & $1.5(1.1)$ & $1.2(1.1)$ \\
\hline Intima media thickness (IMT) & NA & NA & $0.7(0.2)$ & $0.6(0.2)$ & NA \\
\hline Heart Rate (HR) (bpm) & NA & $71.0(26.4)$ & $76.0(5.6)$ & $74.8(5.5)$ & $74.0(8.6)$ \\
\hline \multicolumn{6}{|l|}{ Medication: } \\
\hline$\%$ of antihypertensive treatment & $75.3 \%$ & $0 \%$ & $54.5 \%$ & $0 \%$ & $0 \%$ \\
\hline$\%$ of antilipidemic treatment & $20.2 \%$ & $0 \%$ & $58.4 \%$ & $0 \%$ & $0 \%$ \\
\hline
\end{tabular}

${ }^{1}$ Pooled HYPEST and CADCZ control subjects, who had no personal history of cardiovascular disease, including essential hypertension, myocardial infarction, coronary artery disease, stroke, and had never been prescribed antihypertensive or other cardiovascular medications. Previously, no population differentiation was detected between HYPEST and CADCZ study subjects[18]

${ }^{2}$ Cases: age at the onset of the essential hypertension (HYPEST) or coronary artery disease (CADCZ); Controls: age at the recruitment

$\mathrm{mmHg}$ - millimeters of mercury; bpm - beats per minute; NA - not available

\section{Laboratory measurements}

Altered serum lipid profile is considered as a cardiovascular risk factor - a condition that is associated with an increased risk of developing CVD affecting the heart and blood vasculature. In the current study, lipid measurements (total-cholesterol, HDL-cholestrerol, LDLcholesterol and triglycerides, TG) were determined from fasting venous blood samples in the HYPEST and CADCZ subjects. For HYPEST total-cholesterol, HDLcholesterol, LDL-cholesterol, and triglycerides in the serum were measured by standardized assays (Cobas Integra $800^{\circ}$ analytical platform, Roche Diagnostics, Inc., USA) at the United Laboratories, Tartu University Hospital [20] or at the Diagnostics Division Laboratory, the North Estonia Medical Centre [21]. For CADCZ, serum lipids were measured by standard techniques in Institute of Clinical Chemistry of Vinohrady Faculty Hospital.

\section{Statistical analysis}

For all identified polymorphisms, the deviation from Hardy-Weinberg equilibrium and differences in allele frequencies between populations were tested using an exact test implemented in Genepop web Version 3.4 [22]. Four rare population-specific polymorphisms showed differences in allele frequencies between the studied individuals from HYPEST $(\mathrm{n}=22)$ and CADCZ ( $\mathrm{n}$ = 24) (Fisher's exact test, $\mathrm{p}<0.05$; data not shown). There was no significant difference between the $\operatorname{HYPEST}(\mathrm{n}=1122)$ and CADCZ $(\mathrm{n}=670)$ study groups (Fisher's exact test, $\mathrm{p}>0.05$; data not shown) for the distribution of the $14 \mathrm{bp}$ indel polymorphism.

The significance of the associations between the NCX1 $14 \mathrm{bp}$ indel and cardiovascular traits was tested, and odds ratios/effect sizes and confidence intervals were obtained using linear (quantitative traits) and logistic (case-control analysis) regression analyses implemented in the PLINK software [23]. In all study stages the association analyses were performed under additive and dominant genetic models. Additive genetic models assume a trend per copy of the minor allele to contribute to the trait or disease susceptibility on genotype categories, whereas dominant genetic models assume that heterozygotes have the same increased risk as minor homozygous genotypes. Recessive genetic model was not applied, as it requires a large sample size to reach a reasonable statistical power. Meta-analysis was performed using inverse variance method with fixed effect model of both sample sets. Association tests were performed with age, sex and BMI as co-variates. $P$ values $<0.05$ were considered statistically significant. Two additional corrections were used in the quantitative parameter analysis: in the serum lipid biomarker analysis, a correction according to Jun $\mathrm{Wu}$ was implemented to all of the individuals obtaining lipid-lowering medications [24], and in the blood pressure (SBP and DBP) association test, a correction described by Martin Tobin was used for all subjects receiving antihypertensive treatment [25]. For the multiple comparisons (11 traits 
tested) a simple Bonferroni correction was used ( $\mathrm{p}<$ $0.05 / 11=0.0045$ ).

Multiple sequence alignment of the human, common chimpanzee and rhesus macaque DNA sequences of the orthologous regions adjacent to $14 \mathrm{bp}$ indel within NCX1 intron 2 was performed with web-based analysis tool ClustalW2 [26]. LD structure (based on the HapMap variation data) for the analyzed NCX1 genomic region was performed with the Haploview package [27] (See in additional file 3).

\section{Results}

\section{DHPLC screening of polymorphisms in NCX1 non-coding regions}

We analyzed human cardiovascular candidate gene, $\mathrm{NCX} 1(\mathrm{Na}(+)-\mathrm{Ca}(2+)$ exchanger, 2p22.1), for the conserved non-coding regions using VISTA genome browser [15] with default parameters. In total, we identified 365 conserved non-coding regions between human and mouse and rat (May 2004, NCBI Build 33). These conserved non-coding regions (CNR) covered $15.1 \%$ of the analyzed genomic region (gene $\pm 10 \mathrm{~kb}$ ). Based on the length $(50-300 \mathrm{bp}$ ), location ( $>200 \mathrm{bp}$ from the nearest exon) and sequence conservation $(>70 \%$ between human and both rodents), 29 regions were selected for further polymorphism screening among 46 East-European cardiovascular disease patients (HYPEST $\mathrm{n}=22$; $\mathrm{CADCZ} \mathrm{n}=$ $24)$. Due to the limitations of the technology and/or complex structure of the DNA sequence, 13 of the selected NCX1 regions did not qualify for the DHPLC screening. The remaining 16 regions were subjected to polymorphism discovery among cardiovascular phenotype patients. Detailed descriptions of the selected regions as well as inclusion/exclusion criteria for the analysis are given in additional file 1 . In total, ten genetic variants (including three novel variants) were identified within the 16 analyzed regions (Table 2). Most of the variations were located within the second intron of the gene (first intron relative to ATG initiation codon), including six SNPs, and one 14 bp indel (rs11274804, NCBI dbSNP database). Two SNPs were detected in the NCX1 intron 10 and one SNP in intron 4. Among the screened HYPEST samples $(n=22)$ three common (minor allele frequency, MAF $>10 \%$ ) and four rare $(\mathrm{MAF}<10 \%)$ variants were identified. Five polymorphisms detected in the studied CADCZ patients ( $\mathrm{n}$ $=24)$ were uncommon $(\mathrm{MAF}<10 \%)$ and four were common $(\mathrm{MAF}>10 \%)$. Four variants were specific to one of the studied sample sets (either HYPEST or CADCZ).

\section{Characterization of the intronic hypervariable region}

Nine of the identified variants in screened NCX1 noncoding regions were either singletons or targeted already by commercial platforms and thus included in large number of studies (Table 2). The current study focused on the $14 \mathrm{bp}$ indel (rs11274804), which was represented with substantial frequency (6.82\% in HYPEST; $14.58 \%$ in CADCZ) in both study populations. In addition, the location of rs11274804 indel variant in the second intron of $N C X 1$ (the first intron in the coding region) raised the hypothesis about its potential effect on the gene transcription as several gene expression regulatory

Table 2 Polymorphisms detected by DHPLC in screened human NCX1 non-coding regions

\begin{tabular}{|c|c|c|c|c|c|c|c|}
\hline Analyzed regions & & Detected & variants $^{1}$ & & & & \\
\hline $2^{2}$ Contig positions in Chr. & Length (bp) & Location & Alleles ${ }^{3}$ & $\begin{array}{l}\text { HYPEST } \\
{\text { (hom } / \text { het })^{4}}^{4}\end{array}$ & $\begin{array}{l}\text { CADCZ } \\
{\text { (hom } / \text { het })^{4}}^{4}\end{array}$ & $\begin{array}{l}\text { Validation/ } \\
\text { rs-number }{ }^{2}\end{array}$ & $\begin{array}{l}\text { Targeted by genotyping } \\
\text { platform }^{5}\end{array}$ \\
\hline 40349616 & 293 & intron 2 & $\mathrm{~A} / \mathrm{g}$ & ND & $1 / 4$ & novel & NA \\
\hline $40335702-40335701$ & 348 & intron 2 & 14 bp indel ${ }^{6}$ & $0 / 3$ & $0 / 7$ & rs $11274804^{6}$ & NA \\
\hline 40335650 & 348 & intron 2 & $\mathrm{C} / \mathrm{g}$ & $0 / 1$ & $0 / 1$ & novel & NA \\
\hline 40407194 & 387 & intron 2 & $\mathrm{C} / \mathrm{g}$ & $0 / 1$ & $1 / 3$ & rs72943138 & Illumina \\
\hline 40277948 & 370 & intron 2 & $\mathrm{~T} / \mathrm{C}$ & $3 / 6$ & $0 / 2$ & rs449383 & Affymetrix GeneChip \\
\hline 40475254 & 469 & intron 2 & $\mathrm{~A} / \mathrm{t}$ & $3 / 9$ & $0 / 1$ & rs2192773 & Illumina \\
\hline 40301091 & 292 & intron 2 & $a / G$ & ND & $1 / 1$ & rs2540904 & Illumina \\
\hline 40246617 & 282 & intron 4 & $\mathrm{~T} / \mathrm{C}$ & ND & $0 / 1$ & novel & NA \\
\hline 40514809 & 489 & intron 10 & $\mathrm{~A} / \mathrm{g}$ & $3 / 9$ & $0 / 5$ & rs4952414 & Illumina \\
\hline 40514961 & 489 & intron 10 & $c / T$ & $0 / 1$ & ND & rs17026003 & Affymetrix GeneChip \\
\hline
\end{tabular}

${ }^{1}$ Polymorphism screening was performed using HYPEST $(n=22)$ and CADCZ $(n=24)$ cases. Detailed description of all targeted genomic regions and detected variants is given in additional file 1.

${ }^{2}$ Contig positions and rs-numbers of the validated SNPs are given according to the Simple Nucleotide Polymorphisms database (dbSNP build $129 ;$ Human Genome March 2006).

${ }^{3}$ Major and minor alleles are indicated with capital and lower case letters, respectively.

${ }^{4}$ Number of number of homozygotes/heterozygotes (hom/het) of each identified polymorphism among the screened individuals

${ }^{5}$ Commercial genotyping platforms, which include the described variants (according to NCBI database)

${ }^{6}$ the 14 bp indel: CATTCCCTCTCCAT/-

ND - not detected; NA - not available 
elements have been mapped within the first introns [28-30]. Thus, this polymorphism as well as its flanking region was selected for further characterization.

The region flanking the $14 \mathrm{bp}$ indel (348 bp) in NCX1 intron 2 was targeted for the larger-scale genotyping using Denaturing Gradient Gel Electrophoresis (DGGE). Our study samples, HYPEST $(\mathrm{n}=1122)$ and CADCZ $(\mathrm{n}$ $=670$ ), displayed nine different genotype variants of this intronic segment (representing seven novel alternative alleles), detected by DGGE and confirmed by sequencing (Figure 1). The analyzed region appeared to be highly polymorphic in both sample collections. In addition to $14 \mathrm{bp}$ indel, a SNP (C/G), a duplication of $40 \mathrm{bp}$ segment, and four alternative deletions (10 bp, $43 \mathrm{bp}$ and $5 \mathrm{bp}$ ) were localized within the $348 \mathrm{bp}$ region (Figure 1, Table 3). The breakpoints of several detected variants co-localized with the $14 \mathrm{bp}$ indel (Figure $2 \mathrm{~b}$ ). The allele frequencies of the detected variants in the full genotyped sample $(\mathrm{n}=1792)$ varied from singletons $(10$ bp deletion; $40 \mathrm{bp}$ duplication) to common polymorphisms with allele frequencies up to $8.51 \%$ (14 bp indel). The 43 bp deletion was enriched in HYPEST samples (20 subjects in HYPEST versus 1 in CADCZ). One HYPEST subject appeared to be a compound heterozygote for $14 \mathrm{bp}$ indel/43 bp deletion. In total, $18.21 \%$ of the genotyped HYPEST subjects and $20.49 \%$ of CADCZ subjects were carrying alternative variants of the studied NCX1 intron 2 segment (Table 3). Active genome dynamics of the analyzed region is supported by its location between two LD-blocks within the NCX1 gene (See in additional file 3).

Identified $14 \mathrm{bp}$ indel originates from a human-specific deletion compared to ancestral primate sequence In order to determine the ancestral primate variant of the studied $348 \mathrm{bp}$ region, the consensus sequences of human, common chimpanzee and rhesus macaque were aligned. In contrast to the expectations, multiple sequence alignment (ClustalW2) revealed that the ancestral primate variant is actually the minor human allele carrying the $14 \mathrm{bp}$ sequence motif. The major human variant (among East-European subjects) has evolved through a 14 bp human-specific deletion when compared to sister-species chimpanzee and rhesus macaque (Figure $2 b$ ).

Other identified short insertion-deletion variants within the studied region have occurred in human lineage on the chromosomal variant carrying the humanspecific 14 bp deletion (Figure $2 b$ ).

\section{Association of the $14 \mathrm{bp}$ indel with cardiovascular traits}

Association of NCX1 intronic $14 \mathrm{bp}$ indel (rs11274804) with cardiovascular disease was studied in two Eastern European sample sets: essential hypertension (HYPEST, Estonia; $n=1122$; cases $n=470 /$ controls $=652$ ) and coronary artery disease, CAD (CADCZ, Czech; $\mathrm{n}=670$; cases $n=257 /$ controls $=413)$. Associations were assessed using logistic regression under additive and dominant effect models (age, sex, and BMI as covariates; Table 4). NCX1 intronic 14 bp indel region revealed strong association with the diagnosis of CAD $(P=$ $0.0016, \mathrm{OR}=2.02 ; P=0.0018, \mathrm{OR}=2.07$; additive and dominant models, respectively). As there are various clinical conditions that comprise CAD, additional casecontrol analysis was performed using patients diagnosed with CAD as well as metabolic syndrome $(\mathrm{n}=88)$. Despite a three fold reduction in sample size compared to the full CADCZ patient group, the analysis of CAD patients with metabolic syndrome revealed highly significant association with increased effect size $(P=0.0014$, $\mathrm{OR}=2.34 ; P=0.0016, \mathrm{OR}=2.41$; additive and dominant models, respectively). These associations remained

Table 3 Distribution of insertion/deletion variants identified in the NCX1 intron 2 hypervariable region

\begin{tabular}{|c|c|c|c|c|c|}
\hline & $\begin{array}{l}\text { HYPEST } \\
\text { Cases }\end{array}$ & Controls & $\begin{array}{l}\text { CADCZ } \\
\text { Cases }\end{array}$ & Controls & All \\
\hline Number of studied individuals & 470 & 652 & 257 & 413 & 1792 \\
\hline \multicolumn{6}{|l|}{ Detected genotypes ${ }^{1}(\mathrm{n}, \%)$ : } \\
\hline WT homozygote & $379(80.6 \%)$ & $513(78.7 \%)$ & $192(74.7 \%)$ & $356(86.2 \%)$ & 1440 \\
\hline 14 bp indel heterozygote & $68(14.5 \%)$ & $117(18.9 \%)$ & $55(21.4 \%)$ & $53(12.8 \%)$ & 293 \\
\hline $14 \mathrm{bp}$ indel/C $=>\mathrm{G}$ compound heterozygote & $8(1.7 \%)$ & $8(1.2 \%)$ & $5(1.9 \%)$ & $3(0.7 \%)$ & 24 \\
\hline 14 bp indel homozygote & $3(0.6 \%)$ & 1 & $2(0.8 \%)$ & 1 & 7 \\
\hline $43 \mathrm{bp}$ del heterozygote & $10(2.1 \%)$ & $10(1.5 \%)$ & 1 & 0 & 21 \\
\hline 40 bp duplication & 1 & 0 & 0 & 0 & 1 \\
\hline $14 \mathrm{bp}$ indel/43 bp del compound heterozygote & 0 & 1 & 0 & 0 & 1 \\
\hline 5 bp del heterozygote & 1 & $2(0.3 \%)$ & 1 & 0 & 4 \\
\hline 10 bp del heterozygote & 0 & 0 & 1 & 0 & 1 \\
\hline
\end{tabular}

${ }^{1}$ Exact sequences of detected alleles are given in Figure 2.

$\mathrm{n}=$ number of carriers of the genotype; indel - insertion/deletion; del - deletion 


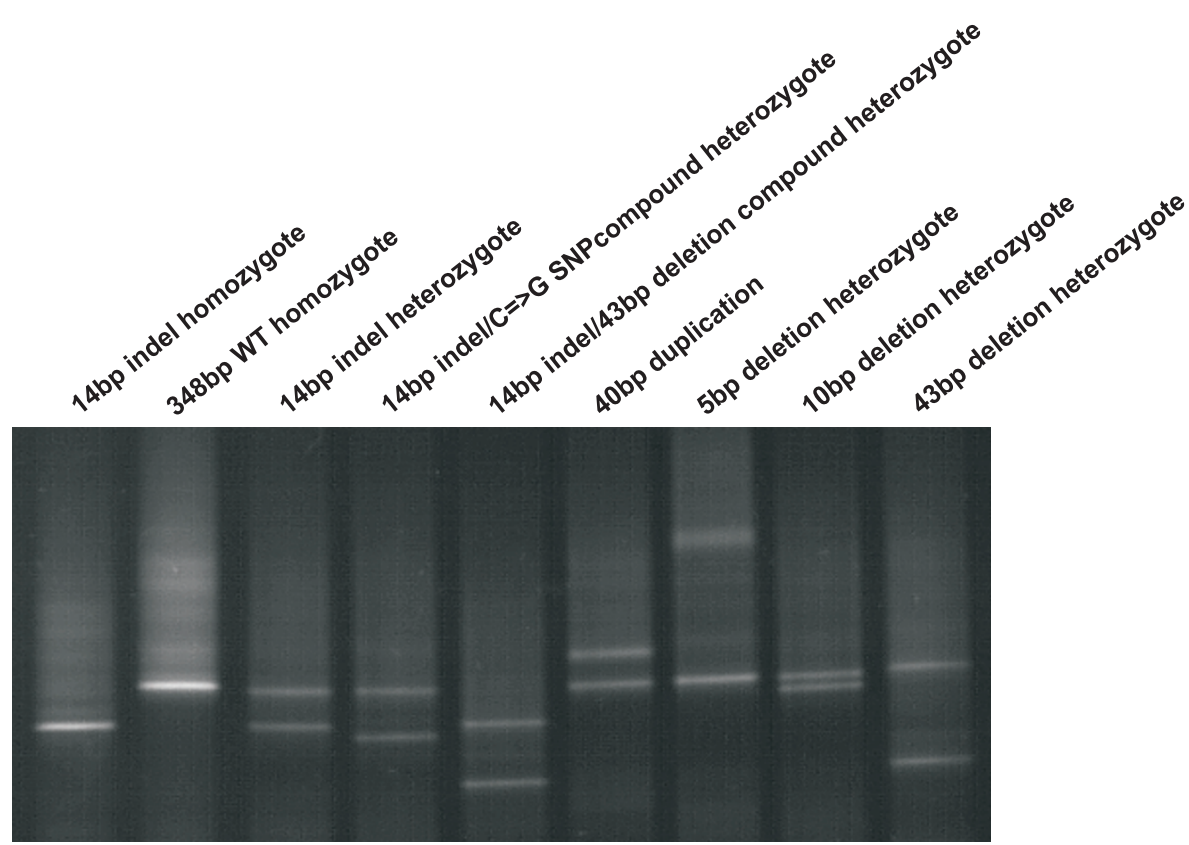

Figure 1 Detection of alternative genotype variants of human NCX1 intron 2 studied region (348 bp) by Denaturing Gradient Gel Eelectorphoresis (DGGE). DGGE was performed using 9\% polyacrylamide gel in $0.5 \times$ TAE buffer containing 30-85\% denaturing gradient of ureumformamide. Electrophoresis conditions were $58^{\circ} \mathrm{C}, 12 \mathrm{~h}$ and $140 \mathrm{~V}$.

significant after correction for multiple testing (Bonferroni significance level $\alpha=0.05 / 11=0.0045$ ).

NCX1 intronic 14 bp indel was not associated with the diagnosis of essential hypertension in HYPEST sample collection $(P>0.1)$.

\section{Association of the $14 \mathrm{bp}$ indel with quantitative cardiovascular parameters}

Associations of the 14 bp indel with quantitative cardiovascular parameters [systolic (SBP) and diastolic (DBP) blood pressure, heart rate, Intima-Media Thickness (IMT)], and serum lipid biomarkers [total cholesterol, HDL, LDL, triglycerides (TG)] were evaluated by linear regression under additive and dominant models (Table 5, Table 6). A marginal negative correlation was detected with heart rate $(P=0.04$, beta $=-1.6$; additive model $)$ and LDL $(P=0.04$, beta $=-0.26$; dominant model) among healthy Eastern-European subjects (Table 5 , Table 6). Association of the $14 \mathrm{bp}$ indel with serum triglyceride levels reached marginal significance in the CADCZ sample $(P=0.04$, beta $=0.25$; both models $)$ and a non-significant trend for association in the joint meta-analysis with HYPEST data $(P=0.08$, beta $=0.18$; $P=0.07$, beta $=0.19$; additive and dominant models, respectively). No significant association was detected with other studied cardiovascular and serum lipid parameters in separate sample sets and in meta-analysis.

\section{Discussion}

We subjected the human $\mathrm{NCX} 1\left(\mathrm{Na}^{+} / \mathrm{Ca}^{2+}\right.$ exchanger $)$ gene to polymorphism screening in conserved non-coding regions with the aim to identify novel potential regulatory variants, which may contribute to the development of cardiovascular disease (CVD). So far, fine-scale polymorphism discovery in the coding, and promoter regions of the NCX1 gene have been carried out only among Japanese individuals, where twopromoter SNPs were shown to be associated with essential hypertension [8] as one of the major risk factor for several CVDs (i.e. CAD). We conducted the polymorphism discovery in NCX1 non-coding conserved regions using CVD patients from two Eastern-European sample collections (HYPEST, essential hypertension; CADCZ, coronary artery disease). Among the ten identified variants, the genomic context of the 14 bp indel located in NCX1 gene intron 2 and its association with CVD was studied in detail. First introns have previously been indicated to contain essential regulatory elements and therefore, may contribute to the transcriptional regulation processes [31] and splicing. For example, in human CFTR gene a regulatory intronic DNase I hypertensive site (DHS) was shown to be required for the normal expression levels in the intestinal epithelium in vivo [28]. The expression profiles of human angiotensin II type 2 receptor AGTR2 and erythroid-specific GATA-1 are affected by regulatory 
elements in intron 1 containing transcription factor binding sites $[29,30]$.

Genotyping of the $14 \mathrm{bp}$ indel locus revealed the hypervariable nature of the studied genomic segment within NCX1 intron 2. Among the screened EasternEuropeans $(\mathrm{n}=1792)$ the analyzed 348 bp region was represented by seven different alleles (Figure 1, Figure 2 ). The alignments of human-chimpanzee-macaque sequences revealed that the major human variant (allele frequency $90.45 \%$ ) was actually a human-specific deletion compared to other primates. The most common alternative variant, the $14 \mathrm{bp}$ indel, appeared to have the ancestral status among primates. Both, chimpanzee and rhesus macaque possess this common 14 bp sequence tract in their $N C X 1$ intron 2 (Figure 2b). Either natural selection or genetic drift may have contributed to the enrichment of the novel $14 \mathrm{bp}$ deletion variant among humans. The data suggests that the novel deletion variant may carry a selective advantage among humans as it was found to be associated with decreased risk for $\mathrm{CAD}$ and elevated serum triglyceride levels. In primate evolution, emergence of such short indel variants and indel-related transcriptional and translational changes may have provided an additional source for the flexible 
Table 4 Association between cardiovascular disease and NCX1 intronic 14 bp indel rs11274804

\begin{tabular}{|c|c|c|c|c|c|c|c|c|}
\hline \multirow[b]{2}{*}{ Disease } & \multirow[b]{2}{*}{ Sample } & \multirow[b]{2}{*}{$\begin{array}{l}\text { Sample size } \\
\text { cases/ } \\
\text { controls }^{2}\end{array}$} & \multicolumn{2}{|c|}{$\begin{array}{l}\text { Minor allele } \\
\text { frequency (\%) }\end{array}$} & \multicolumn{4}{|c|}{$\begin{array}{l}\text { Association testing using logistic regression } \\
\text { Additive model }\end{array}$} \\
\hline & & & Cases & Controls & P-value & OR $[95 \% \mathrm{Cl}]$ & P-value & OR $[95 \% \mathrm{Cl}]$ \\
\hline Essential hypertension & HYPEST & $470 / 652$ & 7.76 & 9.04 & 0.14 & $\begin{array}{l}0.70 \\
{[0.44,1.12]}\end{array}$ & 0.09 & $\begin{array}{l}0.65 \\
{[0.40,1.06]}\end{array}$ \\
\hline Coronary artery disease & CADCZ & $257 / 413$ & 12.45 & 7.02 & 0.0016 & $\begin{array}{l}2.02 \\
{[1.30,3.13]}\end{array}$ & 0.0018 & $\begin{array}{l}2.07 \\
{[1.31,3.26]}\end{array}$ \\
\hline Coronary artery disease \& metabolic syndrome & CADCZ & $88 / 361$ & 15.07 & 7.64 & 0.0014 & $\begin{array}{l}2.34 \\
{[1.38,3.96]}\end{array}$ & 0.0016 & $\begin{array}{l}2.41 \\
{[1.39,4.18]}\end{array}$ \\
\hline
\end{tabular}

${ }^{1}$ Logistic regression analysis was performed with the following covariates: sex, age, BMI.

${ }^{2}$ Detailed definition of cases and controls for essential hypertension, coronary artery disease and metabolic syndrome is given in Materials and Methods, as well as in additional file 2 .

Significant differences have been highlighted in bold, $\mathbf{P}<\mathbf{0 . 0 5}$

response of genomes to the changing life-style and environmental conditions. As a supportive observation, an enrichment of indels in immunity-associated loci has been found as a possible response to variable virus infections (i.e. HIV) in human and chimpanzee [32].

The human-specific deletion variant is surrounded by an abundance of other short (5-43 bp) deletion variants and a duplication (40 bp) polymorphism, which possess overlapping breakpoints (Figure 2b). This observed high variation refers to a potential indel hotspot, which may have been triggered by the initial $14 \mathrm{bp}$ deletion in human lineage. This scenario is consistent with a recent report revealing the mutagenic role of the indel heterozygosity to its surrounding sequences [33]. The state of indel heterozygosity is expected to affect localized chromosome pairing during meiosis. Regions with indel heterozygosity might be prone to double stranded DNA breaks and are thus targeted to mutational repair, which in turn leads to higher mutation rate [33,34]. A consensus sequence motif GTAAG has been reported with the high prevalence within genomic regions prone to insertion/deletion events $[35,36]$. The sequence (CATTCCCTCTCCAT) of the $14 \mathrm{bp}$ indel identified in this study contains the inverted sequence of this previously described hotspot motif on the reverse strand (GTAAG vs. CATTC). In addition, the studied

Table 5 Association between cardiovascular parameters and the carrier status of the 14 bp indel in NCX1 intron 2

\begin{tabular}{|c|c|c|c|c|c|c|c|c|c|}
\hline & \multirow[b]{3}{*}{ Sample } & \multirow[b]{3}{*}{$\mathrm{n}$} & \multirow{3}{*}{$\begin{array}{l}\text { WT/WT } \\
\text { Mean ( } \pm \text { SD) }\end{array}$} & \multirow{3}{*}{$\begin{array}{l}\text { WT/ } \\
\text { indel } \\
\text { Mean ( } \pm \text { SD) }\end{array}$} & \multirow{3}{*}{ 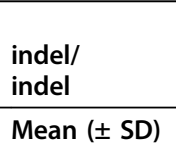 } & \multicolumn{4}{|c|}{ Association testing using linear regression ${ }^{1}$} \\
\hline & & & & & & & ve model & Domi & ant model \\
\hline & & & & & & P-value & Effect (SE) & P-value & Effect (SE) \\
\hline \multirow[t]{4}{*}{$\mathrm{SBP}(\mathrm{mmHg})^{2}$} & HYPEST & 997 & $140.7(21.19)$ & $141.4(22.93)$ & $168.7(36.14)$ & 0.31 & $1.50(1.47)$ & 0.45 & $1.14(1.52)$ \\
\hline & CADCZ & 670 & $132.4(20.29)$ & $134.1(22.00)$ & $135.0(18.03)$ & 0.44 & $1.43(1.85)$ & 0.42 & $1.54(1.92)$ \\
\hline & Meta-analysis & 1667 & $137.4(21.23)$ & $138.4(22.80)$ & $151.8(31.51)$ & 0.20 & $1.47(1.15)$ & 0.28 & $1.29(1.19)$ \\
\hline & Healthy subjects ${ }^{3}$ & 1048 & $128.5(5.66)$ & $128.0(10.53)$ & $136.5(9.19)$ & 0.47 & $-0.71(0.98)$ & 0.39 & $-0.86(0.99)$ \\
\hline \multirow[t]{4}{*}{$\mathrm{DBP}(\mathrm{mmHg})^{2}$} & HYPEST & 993 & $86.9(12.67)$ & $87.2(13.31)$ & $104.3(22.28)$ & 0.39 & $0.82(0.95)$ & 0.57 & $0.55(0.98)$ \\
\hline & CADCZ & 669 & $84.2(11.51)$ & $84.1(15.45)$ & 86.7 (11.55) & 0.71 & $0.39(1.07)$ & 0.73 & $0.38(1.11)$ \\
\hline & Meta-analysis & 1662 & $85.8(12.29)$ & $85.9(14.29)$ & 95.5 (18.59) & 0.38 & $0.63(0.71)$ & 0.51 & $0.48(0.74)$ \\
\hline & Healthy subjects ${ }^{3}$ & 1048 & $81.4(1.41)$ & $80.5(7.85)$ & $85.0(7.07)$ & 0.11 & $-1.06(0.66)$ & 0.08 & $-1.15(0.67)$ \\
\hline \multirow[t]{2}{*}{ Heart rate (bpm) } & CADCZ & 670 & $75.1(5.51)$ & $76.2(5.83)$ & $69.3(3.06)$ & 0.15 & $0.79(0.54)$ & 0.07 & $1.03(0.56)$ \\
\hline & Healthy subjects ${ }^{3}$ & 833 & $75.0(8.49)$ & $73.3(8.06)$ & $70.0(0.00)$ & 0.04 & $-1.61(0.79)$ & 0.05 & $-1.60(0.80)$ \\
\hline \multirow{2}{*}{$\begin{array}{l}\text { IMT } \\
(\mathrm{mm})\end{array}$} & CADCZ & 670 & $0.63(0.20)$ & $0.65(0.20)$ & $0.87(0.29)$ & 0.20 & $0.03(0.02)$ & 0.33 & $0.02(0.02)$ \\
\hline & CADCZ controls & 413 & $0.58(0.17)$ & $0.54(0.15)$ & $1.20(0.00)$ & 0.42 & $-0.02(0.02)$ & 0.18 & $-0.03(0.02)$ \\
\hline
\end{tabular}

${ }^{1}$ For association analysis with SBP and DBP regression testing for a linear trend of marker alleles was performed with age, sex and BMI as covariates. Association analysis with heart rate was performed with sex as covariate, and intima-media thickness without covariates.

${ }^{2}$ Correction for antihypertensive treatment was implemented to all treated patients as described[25].

${ }^{3}$ Pooled HYPEST and CADCZ control subjects, who had no personal history of cardiovascular disease, including essential hypertension, myocardial infarction, coronary artery disease, stroke, and had never been prescribed cardiovascular medications. Previously, no population differentiation was detected between HYPEST and CADCZ study subjects[18]

$\mathrm{n}$ - number of individuals; IMT - Intima-media thickness; Significant differences have been highlighted in bold, $\mathbf{p}<\mathbf{0 . 0 5}$ 
Table 6 Association between serum lipid biomarkers and the carrier status of the 14 bp indel in NCX1 intron 2

\begin{tabular}{|c|c|c|c|c|c|c|c|c|c|}
\hline & \multirow[b]{3}{*}{ Sample ${ }^{2}$} & \multirow[b]{3}{*}{$\mathbf{n}$} & \multirow{3}{*}{$\begin{array}{l}\text { WT/WT } \\
\text { Mean ( } \pm \text { SD) }\end{array}$} & \multirow{3}{*}{$\begin{array}{l}\text { WT/indel } \\
\text { Mean ( } \pm \text { SD) }\end{array}$} & \multirow{3}{*}{$\begin{array}{l}\begin{array}{l}\text { indel/ } \\
\text { indel }\end{array} \\
\text { Mean }( \pm S D)\end{array}$} & \multicolumn{4}{|c|}{ Association testing using linear regression ${ }^{1}$} \\
\hline & & & & & & & & & \\
\hline & & & & & & P-value & Effect (SE) & P-value & Effect (SE) \\
\hline \multirow{4}{*}{$\begin{array}{l}\text { Total cholesterol } \\
(\mathrm{mmol} / \mathrm{L})\end{array}$} & HYPEST & 459 & $5.92(1.15)$ & $5.85(1.24)$ & $6.40(2.47)$ & 0.86 & $-0.02(0.14)$ & 0.73 & $-0.05(0.15)$ \\
\hline & CADCZ & 670 & $5.50(1.04)$ & $5.48(0.99)$ & $5.55(0.83)$ & 0.11 & $0.17(0.11)$ & 0.14 & $0.17(0.11)$ \\
\hline & Meta-analysis & 1129 & $5.67(1.10)$ & $5.63(1.10)$ & $5.98(1.71)$ & 0.25 & $0.10(0.09)$ & 0.33 & $0.09(0.09)$ \\
\hline & Healthy subjects ${ }^{3}$ & 431 & $5.59(1.07)$ & $5.36(0.98)$ & $6.37(0.00)$ & 0.18 & $-0.19(0.14)$ & 0.14 & $-0.22(0.15)$ \\
\hline \multirow[t]{4}{*}{$\mathrm{HDL}(\mathrm{mmol} / \mathrm{L})$} & HYPEST & 458 & $1.52(0.42)$ & $1.46(0.42)$ & $1.38(0.29)$ & 0.23 & $-0.06(0.05)$ & 0.24 & $-0.06(0.05)$ \\
\hline & CADCZ & 670 & $1.41(0.39)$ & $1.41(0.42)$ & $1.42(0.29)$ & 0.81 & $-0.009(0.04)$ & 0.82 & $-0.01(0.04)$ \\
\hline & Meta-analysis & 1128 & $1.45(0.41)$ & $1.43(0.42)$ & $1.40(0.26)$ & 0.35 & $-0.03(0.03)$ & 0.37 & $-0.03(0.03)$ \\
\hline & Healthy subjects ${ }^{3}$ & 431 & $1.52(0.40)$ & $1.61(0.45)$ & $1.70(0.00)$ & 0.10 & $0.09(0.05)$ & 0.10 & $0.09(0.06)$ \\
\hline \multirow[t]{4}{*}{ LDL (mmol/L) } & HYPEST & 459 & $4.04(1.03)$ & $4.04(1.00)$ & $4.66(2.58)$ & 0.73 & $0.04(0.12)$ & 0.88 & $0.02(0.13)$ \\
\hline & CADCZ & 651 & $3.33(0.89)$ & $3.22(0.85)$ & $3.22(0.63)$ & 0.66 & $-0.09(0.20)$ & 0.57 & $-0.12(0.21)$ \\
\hline & Meta-analysis & 1110 & $3.63(1.01)$ & $3.54(1.00)$ & $3.94(1.85)$ & 0.94 & $0.01(0.11)$ & 0.87 & $-0.02(0.11)$ \\
\hline & Healthy subjects ${ }^{3}$ & 427 & $3.41(0.91)$ & $3.13(0.85)$ & $3.94(0.00)$ & 0.06 & $-0.23(0.12)$ & 0.04 & $-0.26(0.13)$ \\
\hline \multirow{4}{*}{$\begin{array}{l}\text { Tri-glycerides } \\
(\mathrm{mmol} / \mathrm{L})\end{array}$} & HYPEST & 458 & $1.77(1.66)$ & $1.82(1.02)$ & $1.29(0.53)$ & 0.99 & $0.003(0.19)$ & 0.89 & $0.03(0.20)$ \\
\hline & CADCZ & 670 & $1.72(1.19)$ & $1.94(1.27)$ & $2.00(1.16)$ & 0.04 & $0.25(0.12)$ & 0.04 & $0.26(0.12)$ \\
\hline & Meta-analysis & 1128 & $1.74(1.40)$ & 1.89 (1.18) & $1.65(0.90)$ & 0.08 & $0.18(0.10)$ & 0.07 & $0.19(0.10)$ \\
\hline & Healthy subjects ${ }^{3}$ & 431 & $1.52(1.12)$ & $1.40(0.81)$ & $1.62(0.00)$ & 0.44 & $-0.11(0.15)$ & 0.42 & $-0.12(0.15)$ \\
\hline
\end{tabular}

${ }^{1}$ For association analysis with serum lipids regression testing for a linear trend of marker alleles was performed with age, sex and BMI as covariates.

${ }^{2}$ Correction for the treatment with lipid-lowering medication was implemented as described[24].

${ }^{3}$ Pooled HYPEST and CADCZ control subjects with available records for serum lipids. The individuals had no personal history of cardiovascular disease, including essential hypertension, myocardial infarction, coronary artery disease, stroke, and had never been prescribed cardiovascular medications. Previously, no population differentiation was detected between HYPEST and CADCZ study subjects[18]

$\mathrm{n}$ - number of individuals; Significant differences have been highlighted in bold, $\mathbf{p}<\mathbf{0 . 0 5}$

hypervariable $N C X 1$ intronic region harboured two further CATTC motifs (Figure 2b). Low LD in this region additionally refers to active genome dynamics (See in additional file 3).

High prevalence of small indels $(<100 \mathrm{bp})$ has been found to be preferably associated with alternatively spliced genes, where partial inclusion and deletion of genic regions may broaden gene expression profiles in different tissue types [32,36]. Among the 12 exons coded by the human NCX1 gene there are six alternative untranslated 5'-exons denoted 1a-1f. Different combinations of these six exons may result in up to 32 different $\mathrm{Na}^{+} / \mathrm{Ca}^{2+}$ exchanger mRNA transcripts [37]. The hypothesis that preferences in splice-site selection among $N C X 1$ exons $1 \mathrm{a}-1 \mathrm{f}$ (and other alternative exons) may be affected by the genetic composition in the hypervariable intron 2 requires experimental proof.

The knowledge about the involvement of small indels $(<100 \mathrm{bp})$ in increasing/decreasing susceptibility to the development of complex disease is still scarce. A wellknown example is $32 \mathrm{bp}$ deletion in human the CCR5 gene that results in a frameshift and premature termination $[38,39]$. This variant, which is common among Europeans (MAF 9.2\%) and almost absent among Africans contributes to the resistance against HIV-1 infection. It was speculated that a $10 \mathrm{bp}$ direct repeat that flanks the deleted region promoted a recombination event leading to the 32 bp deletion [39]. The effect of indels in noncoding regions is less understood. A recent study identified a 25 bp deletion in intron 32 of the human $M Y B P C 3$ gene leading to the loss of exon 33. The carriers of this deletion develop heritable cardiomyopathies and have increased risk of heart failure in Indian populations [40].

The current study identified a strong association between the carrier status of NCX1 intronic $14 \mathrm{bp}$ indel and increased risk to coronary artery disease (CAD) in the East European population. The risk was the highest among the CAD patients with metabolic disease. Consistent with the effect on metabolic processes, the $14 \mathrm{bp}$ indel was also associated with serum triglyceride levels. Several recent studies have highlighted the role of metabolic factors and metabolic syndrome in determining the extent of CAD and the risk for new vascular events $[41,42]$. In perspective, the association study of rs11274804 as well as other identified indel variants in $N C X 1$ intron 2 with CAD and related metabolic factors is to be extended to other populations. The expression of NCX1 alternative transcripts in vascular muscle (NCX1.3 isoform) and in heart (NCX1.1 isoform) further supports the potential involvement of NCX1 genetic variants in susceptibility 
to coronary artery disease [7]. Functional studies would reveal whether the carrier status of alternative $N C X 1$ intronic indel variants affects the alternative transcript profile of the gene.

\section{Conclusions}

In summary, compared to SNPs targeted by large number of locus-specific and genome-wide assays, considerably less attention has been paid to short insertion-deletions (indels) variants in the human genome. The data of genome dynamics, mutation rate and population genetics of short indels, as well as their impact on gene expressional profile and human disease susceptibility is limited. The characterization of NCX1 intronic hypervariable region enriched in human-specific indel variants contributes to this gap of knowledge.

\section{Additional file 1: Characteristics of analyzed DHPLC and DGGE}

regions. All analyzed $N C X 1$ gene regions for DHPLC and DGGE assays.

Characteristics of 29 regions: their location, conservation, PCR primers

and product length, found polymorphisms and the reason for exclusion

form the study.

Click here for file

[http://www.biomedcentral.com/content/supplementary/1471-2350-1115-S1.XLS]

Additional file 2: Additional information for Materials and Methods. Additional information for Materials and Methods.

Click here for file

[http://www.biomedcentral.com/content/supplementary/1471-2350-11-

15-S2.PDF ]

Additional file 3: LD structure of the human NCX1 gene 348 bp region. $L D$ structure of the human $N C X 1$ gene region (chr2; position: 40 241 046-40 661 226) shown as $r^{2}$-blot. Upper white bar marks the positions of HapMap SNPs. Both arrows indicate the location of $14 \mathrm{bp}$ indel (rs11274804) between two LD-blocks in the second intron of the NCX1 gene.

Click here for file

[http://www.biomedcentral.com/content/supplementary/1471-2350-1115-S3.PDF ]

\section{Acknowledgements}

We express our appreciation to all the HYPEST and the CADCZ participants. We thank Dr. Bohumila Janosikova for technical assistance in recruitment of the CADCZ sample collection. We thank the personnel of the Centre of Cardiology, North Estonia Medical Centre and the Department of Cardiology, University of Tartu for assistance in subject recruitment and data collection for the Estonian HYPEST sample collection.

The study was supported by Wellcome Trust International Senior Research Fellowships (grant no. 070191/Z/03/Z, to ML; no. 070255/Z/03/Z to VK) in Biomedical Science in Central Europe. Additionally, the study has been supported by Estonian Ministry of Education and Science core grant no. 0182721 s06, HHMI International Scholarship \#55005617 (to ML), Estonian Science Foundation grants no ETF7471, travel-stipend from Kristjan-Jaak foundation (KK), and by an institutional Research project from Ministry of Education of the Czech Republic MSM0021620806 (to VK).

\section{Author details}

IInstitute of Molecular and Cell Biology, University of Tartu, Tartu, Estonia. ${ }^{2}$ Centre of Cardiology, North Estonia Medical Centre, Tallinn, Estonia. ${ }^{3}$ Tallinn University of Technology, Department of Biomedical Engineering, Chair of Medical Physics, Tallinn, Estonia. ${ }^{4}$ Department of Cardiology, University of Tartu, Tartu, Estonia. ${ }^{5}$ Department of Medical Sciences, Uppsala University
Hospital, Uppsala, Sweden. ' Institute of Inherited Metabolic Diseases, Charles University - First Faculty of Medicine, Prague, Czech Republic.

\section{Authors' contributions}

KK contributed to the study design, performed polymorphism screening, genotyping and resequencing experiments, contributed to the analysis and interpretation of the data and write the first draft of the manuscript. EO was responsible for the collection of the HYPEST study sample, contributed to the analysis and interpretation of the data, and manuscript writing. SS contributed to the analysis, and manuscript writing. PK was responsible for preparing genomic DNAs of HYPEST and CADCZ study subjects, and assisted in DGGE genotyping. MV and GV contributed to the recruitment of HYPEST essential hypertension patients along with relevant clinical and epidemiological data. NT conducted the VISTA analysis. PJ and MP assisted in collection of HYPEST individuals. AK contributed to the DHPLC polymorphism screening. VK coordinated the recruitment of CADCZ study subjects. ML directed the study design and performance, the recruitment of HYPEST study samples and finalized the manuscript. All authors read and approved the final manuscript.

\section{Competing interests}

The authors declare that they have no competing interests.

Received: 6 October 2009

Accepted: 28 January 2010 Published: 28 January 2010

\section{References}

1. Mohlke KL, Boehnke M, Abecasis GR: Metabolic and cardiovascular traits: an abundance of recently identified common genetic variants. Hum Mol Genet 2008, 17(R2):R102-108.

2. Delles C, McBride MW, Padmanabhan S, Dominiczak AF: The genetics of cardiovascular disease. Trends Endocrinol Metab 2008, 19(9):309-316.

3. Dermitzakis ET, Reymond A, Antonarakis SE: Conserved non-genic sequences - an unexpected feature of mammalian genomes. Nat Rev Genet 2005, 6(2):151-157.

4. Drake JA, Bird C, Nemesh J, Thomas DJ, Newton-Cheh C, Reymond A, Excoffier L, Attar H, Antonarakis SE, Dermitzakis ET, et al: Conserved noncoding sequences are selectively constrained and not mutation cold spots. Nat Genet 2006, 38(2):223-227.

5. Blaustein MP: Physiological effects of endogenous ouabain: control of intracellular Ca2+ stores and cell responsiveness. Am J Physiol 1993, 264(6 Pt 1):C1367-1387.

6. Schillinger W, Fiolet JW, Schlotthauer K, Hasenfuss G: Relevance of Na +-Ca2+ exchange in heart failure. Cardiovasc Res 2003, 57(4):921-933.

7. Iwamoto T, Kita S, Zhang J, Blaustein MP, Arai Y, Yoshida S, Wakimoto K, Komuro I, Katsuragi T: Salt-sensitive hypertension is triggered by $\mathrm{Ca} 2+$ entry via $\mathrm{Na}+/ \mathrm{Ca} 2+$ exchanger type-1 in vascular smooth muscle. Nat Med 2004, 10(11):1193-1199.

8. Kokubo $\mathrm{Y}$, Inamoto N, Tomoike H, Kamide K, Takiuchi S, Kawano Y, Tanaka C, Katanosaka Y, Wakabayashi S, Shigekawa M, et al: Association of genetic polymorphisms of sodium-calcium exchanger 1 gene, NCX1, with hypertension in a Japanese general population. Hypertens Res 2004, 27(10):697-702.

9. Iwamoto T, Kita S, Uehara A, Imanaga I, Matsuda T, Baba A, Katsuragi T: Molecular determinants of $\mathrm{Na}+/ \mathrm{Ca} 2+$ exchange (NCX1) inhibition by SEA0400. J Biol Chem 2004, 279(9):7544-7553.

10. Kraev A, Chumakov I, Carafoli E: The organization of the human gene NCX1 encoding the sodium-calcium exchanger. Genomics 1996, 37(1):105-112.

11. Quednau BD, Nicoll DA, Philipson KD: Tissue specificity and alternative splicing of the $\mathrm{Na}+/ \mathrm{Ca} 2+$ exchanger isoforms NCX1, NCX2, and NCX3 in rat. Am J Physiol 1997, 272(4 Pt 1):C1250-1261.

12. Dunn J, Elias CL, Le HD, Omelchenko A, Hryshko LV, Lytton J: The molecular determinants of ionic regulatory differences between brain and kidney $\mathrm{Na}+/ \mathrm{Ca} 2+$ exchanger (NCX1) isoforms. J Biol Chem 2002, 277(37):33957-33962.

13. Hurtado C, Prociuk M, Maddaford TG, Dibrov E, Mesaeli N, Hryshko LV, Pierce GN: Cells expressing unique $\mathrm{Na}+/ \mathrm{Ca} 2+$ exchange (NCX1) splice variants exhibit different susceptibilities to Ca2+ overload. Am J Physiol Heart Circ Physiol 2006, 290(5):H2155-2162. 
14. Ander BP, Hurtado C, Raposo CS, Maddaford TG, Deniset JF, Hryshko LV, Pierce GN, Lukas A: Differential sensitivities of the NCX1.1 and NCX1.3 isoforms of the $\mathrm{Na}+\mathrm{Ca} 2+$ exchanger to alpha-linolenic acid. Cardiovas Res 2007, 73(2):395-403.

15. Web-based VISTA software. http://genome.lbl.gov/vista/index.html.

16. University of California Santa Cruz (UCSC) Genome Browser. http:// genome.ucsc.edu/.

17. Janosikova B, Pavlikova M, Kocmanova D, Vitova A, Vesela K, Krupkova L, Kahleova R, Krijt J, Kraml P, Hyanek J, et al: Genetic variants of homocysteine metabolizing enzymes and the risk of coronary artery disease. Mol Genet Metab 2003, 79(3):167-175.

18. Kepp K, Juhanson P, Kozich V, Ots M, Viigimaa M, Laan M: Resequencing PNMT in European hypertensive and normotensive individuals: no common susceptibilily variants for hypertension and purifying selection on intron 1. BMC medical genetics 2007, 8:47.

19. International Diabetes Federation. http://www.idf.org/.

20. United Laboratories, Tartu University Hospital. http://www.kliinikum.ee/ verekeskus/.

21. Diagnostics Division Laboratory, the North Estonia Medical Centre. http://www.regionaalhaigla.ee/?op=body\&id=50/.

22. Genepop web Version 3.4. http://genepop.curtin.edu.au/.

23. PLINK software. http://pngu.mgh.harvard.edu/ purcell/plink/.

24. Wu J, Province MA, Coon H, Hunt SC, Eckfeldt JH, Arnett DK, Heiss G, Lewis CE, Ellison RC, Rao DC, et al: An investigation of the effects of lipidlowering medications: genome-wide linkage analysis of lipids in the HyperGEN study. BMC Genet 2007, 8:60.

25. Tobin MD, Sheehan NA, Scurrah KJ, Burton PR: Adjusting for treatment effects in studies of quantitative traits: antihypertensive therapy and systolic blood pressure. Stat Med 2005, 24(19):2911-2935.

26. Web-based analysis tool ClustalW2. http://www.ebi.ac.uk/Tools/clustalw2/ index.html.

27. Haploview software. http://www.broadinstitute.org/haploview/haploview/.

28. Rowntree RK, Vassaux G, McDowell TL, Howe S, McGuigan A, Phylactides M, Huxley C, Harris A: An element in intron 1 of the CFTR gene augments intestinal expression in vivo. Hum Mol Genet 2001, 10(14):1455-1464.

29. Seshasayee D, Geiger JN, Gaines P, Wojchowski DM: Intron 1 elements promote erythroid-specific GATA-1 gene expression. J Biol Chem 2000, 275(30):22969-22977.

30. Warnecke C, Willich T, Holzmeister J, Bottari SP, Fleck E, Regitz-Zagrosek V: Efficient transcription of the human angiotensin II type 2 receptor gene requires intronic sequence elements. Biochem J 1999, 340(Pt 1):17-24.

31. Eddy J, Maizels $\mathrm{N}$ : Conserved elements with potential to form polymorphic G-quadruplex structures in the first intron of human genes. Nucleic Acids Res 2008, 36(4):1321-1333.

32. Chen FC, Chen CJ, Li WH, Chuang TJ: Human-specific insertions and deletions inferred from mammalian genome sequences. Genome Res 2007, 17(1):16-22.

33. Tian D, Wang $Q$, Zhang $P$, Araki H, Yang S, Kreitman M, Nagylaki T, Hudson R, Bergelson J, Chen JQ: Single-nucleotide mutation rate increases close to insertions/deletions in eukaryotes. Nature 2008 455(7209):105-108.

34. Lercher MJ, Hurst LD: Human SNP variability and mutation rate are higher in regions of high recombination. Trends Genet 2002, 18(7):337-340.

35. Ball EV, Stenson PD, Abeysinghe SS, Krawczak M, Cooper DN, Chuzhanova NA: Microdeletions and microinsertions causing human genetic disease: common mechanisms of mutagenesis and the role of local DNA sequence complexity. Hum Mutat 2005, 26(3):205-213.

36. Chuzhanova NA, Anassis EJ, Ball EV, Krawczak M, Cooper DN: Meta-analysis of indels causing human genetic disease: mechanisms of mutagenesis and the role of local DNA sequence complexity. Hum Mutat 2003, 21(1):28-44.

37. Kofuji P, Lederer WJ, Schulze DH: Mutually exclusive and cassette exons underlie alternatively spliced isoforms of the $\mathrm{Na} / \mathrm{Ca}$ exchanger. $J \mathrm{Biol}$ Chem 1994, 269(7):5145-5149.

38. Liu R, Paxton WA, Choe S, Ceradini D, Martin SR, Horuk R, MacDonald ME, Stuhlmann H, Koup RA, Landau NR: Homozygous defect in HIV-1 coreceptor accounts for resistance of some multiply-exposed individuals to HIV-1 infection. Cell 1996, 86(3):367-377.

39. Samson M, Libert F, Doranz BJ, Rucker J, Liesnard C, Farber CM, Saragosti S, Lapoumeroulie C, Cognaux J, Forceille C, et al: Resistance to HIV-1 infection in caucasian individuals bearing mutant alleles of the CCR-5 chemokine receptor gene. Nature 1996, 382(6593):722-725.

40. Dhandapany PS, Sadayappan S, Xue Y, Powell GT, Rani DS, Nallari P, Rai TS, Khullar M, Soares P, Bahl A, et al: A common MYBPC3 (cardiac myosin binding protein $\mathrm{C}$ ) variant associated with cardiomyopathies in South Asia. Nat Genet 2009, 41(2):187-191.

41. Kasai T, Miyauchi K, Kubota N, Tamura H, Kojima T, Yokoyama K, Kurata T, Daida $\mathrm{H}$ : The relationship between the metabolic syndrome defined by various criteria and the extent of coronary artery disease. Atherosclerosis 2008, 197(2):944-950

42. Wassink AM, Graaf van der Y, Olijhoek JK, Visseren FL: Metabolic syndrome and the risk of new vascular events and all-cause mortality in patients with coronary artery disease, cerebrovascular disease, peripheral arterial disease or abdominal aortic aneurysm. Eur Heart J 2008, 29(2):213-223.

43. Krawczak M, Cooper DN: Gene deletions causing human genetic disease: mechanisms of mutagenesis and the role of the local DNA sequence environment. Hum Genet 1991, 86(5):425-441.

Pre-publication history

The pre-publication history for this paper can be accessed here:http://www biomedcentral.com/1471-2350/11/15/prepub

doi:10.1186/1471-2350-11-15

Cite this article as: Kepp et al:: Hypervariable intronic region in NCX1 is enriched in short insertion-deletion polymorphisms and showed association with cardiovascular traits. BMC Medical Genetics 2010 11:15.

\section{Submit your next manuscript to BioMed Central and take full advantage of:}

- Convenient online submission

- Thorough peer review

- No space constraints or color figure charges

- Immediate publication on acceptance

- Inclusion in PubMed, CAS, Scopus and Google Scholar

- Research which is freely available for redistribution

Submit your manuscript at www.biomedcentral.com/submit
C Biomed Central 